\title{
Estudio analítico de la portada de la iglesia de Santiago de Guadix, Granada
}

\author{
Analytical study of the main door of Santiago church, Guadix, \\ Granada
}

\author{
JESÚS ESPINOSA GAITÁN, FRANCISCO GUTIERREZ MONTERO y ROSARIO VILLEGAS SȦNCHEZ \\ Insto. del Patrimonio Histórico. Consejería de Cultura. Junta de Andalucía
}

Fecha de recepción: 16-VI-95

ESPAÑA

\begin{abstract}
RESUMEN
El estudio analitico recogido en el presente articulo se realiza como respuesta a la petición formulada por el párroco de la iglesia de Santiago, D. José $M^{a}$ Ballesteros, a través de la Delegación Provincial de Cultura de Granada. Ante el estado de deterioro de la portada, se solicita al Instituto Andaluz del Patrimonio Histórico un asesoramiento técnico sobre la posible intervención que debería llevarse a cabo para solucionar los problemas que presenta la misma. Dentro de dicho asesoramiento se han realizado los estudios previos necesarios para determinar las caracteristicas de la piedra utilizada, las posibles causas de deterioro y evaluar los productos de tratamiento más adecuados, en caso de que se considere conveniente la aplicación de alguno como parte de la intervención de conservación-restauración.
\end{abstract}

En una primera fase se ha llevado a cabo la inspección visual de la portada, asi como del interior de la iglesia, y posteriormente se ha procedido a la toma de muestras. Dichas muestras se han estudiado por medio de: Análisis Quimico de componentes mayoritarios, Difracción de Rayos $X$, Estudio Petrográfico mediante microscopia óptica y observación al SEM con microsonda de Rayos X. De los resultados se han podido deducir las posibles causas y mecanismos de alteración.

Como paso previo a la evaluación de los tratamientos, y dada la necesidad de utilizar gran cantidad de material pétreo para la realización de los ensayos requeridos, se ha procedido a la localización de la cantera de origen de la piedra, que se halla situada en Bácor, pequeña localidad del término municipal de Guadix a unos $40 \mathrm{~km}$ de ésta. Una vez comprobada la coincidencia con la piedra de la portada, se ha extraido suficiente material para fabricar las probetas necesarias. El estudio de este material pétreo se ha considerado muy interesante por el hecho adicional de que se ha utilizado profusamente también en la Catedral de Guadix, de forma que los resultados que se obtengan en los ensayos podrán ser extensivos también a dicho edificio.

\section{$S U M M A R Y$}

The analytical study described on this paper has been made due to the request formulated by the priest of Santiago church, D. Jose $M^{a}$ Ballesteros, through the Provincial Delegation of Culture of Granada. In view of the degradation of the main door front, the Instituto Andaluz del Patrimonio Histórico has been asked for technical advise on the possible treatment to be carried out to resolve the problems of this front. As a part of the technical study we have carried out the analysis required to determine the characteristics of the stone used, the possible causes of weathering and to evaluate the most adecuate treatment products, in case that it is convenient to apply any one as a part of the conservation restoration works.

As a first step we have carried out a visual inspection of the door front and the inside of the church, and then samples have been taken. These samples have been analyzed by means of: chemical analysis of main components, X Ray difraction, mineralogic petrographic study and SEM observation. From all these determinations it has been deduced the possible causes and mechanisms of alteration.

As previous phase to the evaluation of treatments, due to the high quantity of stone needed to make all the tests, we have proceeded to identify and find the quarry of origin of this stone; it is located in Bácor, a little village on the municipal term of Guadix, at $40 \mathrm{Km}$ from it. Once the identification has been made with certainty, enough material has been extracted to prepare the samples used. It is very interesting to study this stone because it has been employed also on the Cathedral of Guadix and it will be able to extend the results obtained on the tests to this building. 


\section{ANTECEDENTES HISTÓRICOS}

La iglesia de Santiago es el resultado de la transformación de la precedente mezquita musulmana, que a su vez resulta de la transformación de otro precedente templo cristiano, mozárabe, que desaparecería en el siglo XIII (1). Su construcción se sitúa en la primera mitad del siglo XVI, y la portada que aquí se estudia se levantaría en la década de 1540 , posiblemente diseñada por Diego de Siloé.

La portada consta de un cuerpo coronado por un remate. La parte inferior del cuerpo se estructura con una puerta situada entre pilastras laterales con basa y friso en las que se encajan, en cada lado, dos hornacinas superpuestas, actualmente vacías. $\mathrm{El}$ conjunto se remata con un entablamento que sirve de base al remate superior. Este se constituye con una hornacina central, actualmente vacía y que debió cobijar al titular Santiago, ubicada entre relieves dispuestos oblicuamente. Se corona en la parte central con un heráldico imperial con el águila bicéfala de Carlos V, y a ambos lados en medio de los paños, heráldicas del obispo D. Antonio del Aguila (figura 1).

\section{DESCRIPCIÓN DEL ESTADO DE CONSERVACIÓN}

La portada está situada en la fachada Noroeste de la iglesia, abierta a la plaza de Santiago, peatonal en la parte más cercana a la iglesia, siendo el tráfico de vehículos en las calles adyacentes relativamente escaso.

El principal problema que presenta, no sólo la portada sino toda la iglesia, es la humedad ascendente. El alto nivel freático natural, unido a problemas en las redes de saneamiento de los edificios próximos, provocan que la humedad alcance alturas de 2 a 3 metros en los muros y pilares de la iglesia, como se observa en la figura 2.

Debido a esta subida capilar, la piedra de la portada se encuentra mucho más alterada en la parte inferior. En esta zona aparece alveolización en todos sus grados y arenización, hasta provocar pérdidas muy acusadas de los relieves (figura 3), resultando algunos sillares prácticamente destruidos (figura 4). Como consecuencia de esta circulación de agua con sales disueltas y su evaporación en la superficie, aparecen abundantes eflorescencias. Hasta una altura de 1,5 metros aproximadamente (hacia la mitad de las hornacinas), son éstos los indicadores de alteración más acusados.

Por encima de esta altura, donde no llega la humedad ascendente, la piedra aparece cubierta de una costra oscura bastante uniforme y muy dura, que se desprende

\section{HISTORICAL ANTECEDENTS}

Santiago church results of the transformation of a precedent moslem mosque, that, in its turn, ocuppied the place of a christian church, mozarabic, that dissappeared on XIII century (1). The construction of Santiago church took place on the first half of XVI century, and the front door here studied on the decade of 1540, possibly designed by Diego de Siloe (figure 1).

The gate has a single body topped by a cresting. The lower part of the body consists of a door situated between lateral pillars with base and frieze, in which two superimposed niches, empty at present, are fitted on either side. The whole is crowned by an entablature which serves as a basis for the top cresting. The cresting consists of a central niche, empty at present, which must have contained a statue of Santiago, between obliquely arranged reliefs. The central part is crowned by an imperial heraldry with the bicephalous eagle of Carlos $V$, and the heraldries of the bishop Antonio del Aguila in the middle of the walls on either side (Figure 1).

\section{PRESENT STATE OF CONSERVATION}

The door front is situated on the NW facade of the church, open to Santiago square, without car passing on the surroundings of the church and with low density of traffic on adjacent streets.

The main problem that appears, not only on the door front but on the whole building, is raising damp. The high ground water level, besides problems on drainage systems of near buildings, provoke that humidity reaches 2 to 3 metres of height on walls and columns of the church, as can be seen on figure 2 .

Due to this raising damp, the stone of the door is much more weathered on the lower part. In this zone it appears alveolization in all degrees and arenization, that cause severe losses of relieves (figure 3), resulting in some blocks completely destroyed (figure 4). As consequence of the movement of water with dissolved salts and its evaporation on the surface, there are a lot of efflorescences. Up to a height of 1,5 metres approximately (the middle of the niches) these are the clearest weathering indicators.

Over this zone, where rising damp does not arrive, the stone appears covered by a dark crust quite uniform and hard, that falls down locally on most exposed parts 
puntualmente y en las zonas más expuestas y deja al descubierto la piedra que está ligeramente decohesionada. En algunas zonas se detecta la existencia de películas superficiales de colores distintos a la costra ya mencionada, siendo más abundante la presencia de zonas de color rojizo con apariencia de ser restos de policromía. Estos restos son más numerosos en el interior de las hornacinas situadas a ambos lados de la puerta, donde se encuentran más protegidos. En la figura 5 se aprecia la costra oscura que cubre prácticamente toda la portada, con algunas áreas de color rojizo.

En la cornisa aparecen algunas fisuras y rotura de pequeños trozos, mientras que la parte superior presenta los mismos indicadores de alteración de las zonas bajas pero menos pronunciados. Se observan fenómenos de arenización, alveolización, desplacación de la costra oscura, que aparece menos continua que en la parte intermedia al estar menos protegida, y desprendimiento de algunos elementos decorativos. La piedra está menos disgregada que en la zona baja, conservándose mejor las formas de los relieves (figura 6).

\section{TOMA DE MUESTRAS}

La localización de las muestras aparece en la figura 7 y su descripción en la Tabla 1.

\section{DETERMINACIONES REALIZADAS}

\subsection{Estudio Petrográfico}

La piedra utilizada en la portada corresponde toda al mismo litotipo. En corte fresco la piedra presenta un color beige cremoso, textura típica de una calcarenita, y algunos restos fósiles de lamelibranquios identificables a simple vista.

Por observación al microscopio óptico de luz polarizada se ha profundizado en la caracterización del material pétreo.

La roca presenta una textura heterogranular, compuesta mayoritariamente por restos calcáreos de origen fosilífero, formando el entramado principal. Estos restos son, por orden de abundancia, Foraminíferos bentónicos (Nummulites) y algunos Foraminiferos planctónicos (Globutruncanas), y en cantidades mucho menores algunos fragmentos de Bivalvos. A su vez, presenta grandes proporciones de componentes terrígenos subredondeados, salteados homogéneamente por toda la roca, prácticamente todos de cuarzo, apareciendo en menor cantidad algunos feldespatos. En la figura 8 aparece la microfotografia de la lámina delgada. and leaves uncovered the stone that is slightly decohesionated. On some parts there are superficial layers of different colours, beeing most abundant reddish zones that seem to be rests of polychromy. These rests are more numerous on the interior of the niches on both sides of the door, where they are more protected. On figure 5 the dark crust that covers almost all the front is shown and the reddish areas are marked.

On the cornice there are some fisures and cracking of little pieces, while in the upper part the same weathering indicators than in the lower zone are present, but less pronounced, in this case due to the rain water. There are arenization, alveolization, deplacation of dark crust, which is less continue that in the middle zone because it is less protected, and falling down of some decorative elements. The stone is less disagregated that in the lower part, beeing better preserved the relieves (figure 6).

\section{SAMPLES TAKEN}

Samples locations appear on figure 7 and their descriptions on Table 1.

\section{MEASUREMENTS}

\section{1. Petrographical Study}

The stone used on the door front corresponds all to the same lythotype. By visual observation (on fresh cut) it is of beige colour and has tipycal calcarenite texture. It can be seen also some fossils rests of bivalves, which indicates that the stone has origin on bowl edge.

By means of observation with optical microscope with polarized light the material has been studied.

The stone has a heterogranular texture, composed mainly of calcitic compounds of fossil origin that form the main truss. These rests are, in order of importance, bentonic foraminifera (possibly Nummulites), some planctonic foraminifera (Globotruncanes) and, in very smaller quantities, some macrofossils (bivalves). It also has great porportion of terrigenous clasctics quite subrounded, distributed homogeneously on all the stone, almost all of quartz, with little quantities of feldspar. On figure 8 appears a microphotography of thin section. 
TABLA 1 (TABLE 1)

Muestras tomadas (Samples taken)

\begin{tabular}{|c|c|}
\hline Muestra/Sample & Descripción/Description \\
\hline GPS1 & Piedra no alterada (parte interna) \\
\hline & Unweathered stone (inner part) \\
\hline GPS2 & $\begin{array}{l}\text { Escama de piedra despegada } \\
\text { Detached scale of stone }\end{array}$ \\
\hline GPS3 & Costra endurecida \\
\hline & Dark crust \\
\hline GPS4 & Costra endurecida \\
\hline & Dark crust \\
\hline GPS5 & Costra roja \\
\hline & Red layer \\
\hline GPS6 & $\begin{array}{l}\text { Mortero } \\
\text { Mortar }\end{array}$ \\
\hline GPS7 & $\begin{array}{l}\text { Eflorescencias sobre la piedra } \\
\text { Eflorescences over the stone }\end{array}$ \\
\hline GPS8 & $\begin{array}{l}\text { Piedra con costra amarilla } \\
\text { Stone with yellow layer }\end{array}$ \\
\hline GPS9 & $\begin{array}{l}\text { Costra Roja } \\
\text { Red layer }\end{array}$ \\
\hline GPS10 & $\begin{array}{l}\text { Piedra desprendida } \\
\text { Detached piece of stone }\end{array}$ \\
\hline GPS11 & $\begin{array}{l}\text { Piedra alterada } \\
\text { Weathered stone }\end{array}$ \\
\hline GPS12 & $\begin{array}{l}\text { Costra roja } \\
\text { Red layer }\end{array}$ \\
\hline GPS13 & $\begin{array}{l}\text { Piedra } \\
\text { Stone }\end{array}$ \\
\hline GPS14 & $\begin{array}{l}\text { Piedra con liquenes } \\
\text { Stone with lichens }\end{array}$ \\
\hline GPS15 & $\begin{array}{l}\text { Mortero } \\
\text { Mortar }\end{array}$ \\
\hline GPS16 & $\begin{array}{l}\text { Piedra con liquenes } \\
\text { Stone with lichens }\end{array}$ \\
\hline GPS17 & $\begin{array}{l}\text { Piedra con costra } \\
\text { Weathered stone }\end{array}$ \\
\hline GPS18 & $\begin{array}{l}\text { Mortero con costra naranja } \\
\text { Mortar with reddish crust }\end{array}$ \\
\hline GPS19 & $\begin{array}{l}\text { Piedra desprendida } \\
\text { Detached piece of stone }\end{array}$ \\
\hline GPS20 & $\begin{array}{l}\text { Piedra con costra oscura } \\
\text { Stone with dark crust }\end{array}$ \\
\hline GPS21 & $\begin{array}{l}\text { Piedra con costra naranja } \\
\text { Stone with orange crust }\end{array}$ \\
\hline GPS22 & $\begin{array}{l}\text { Piedra con costra } \\
\text { Stone with dark crust }\end{array}$ \\
\hline
\end{tabular}


La roca se clasificaría como una biocalcarenita micrítica, con facies de foraminíferos bentónicos y planctónicos, con gran abundancia de terrígenos; facies típica del Subbético medio y de origen de borde de cuenca.

La porosidad es muy elevada, la cual se ha visto incrementada respecto a la original de la roca en su génesis por la disolución de las cámaras de las globutruncanas. Los poros, además, se encuentran intercomunicados, lo que facilita la circulación de fluidos en su interior.

También se observan al microscopio recristalizaciones de carbonato cálcico micrítico en los bordes de los poros, lo cual indica que, efectivamente, ha habido circulación de fluidos que han disuelto el carbonato original, que ha reprecipitado posteriormente en los poros.

Los terrígenos feldespáticos se presentan bastante alterados a fases más hidratadas, como son minerales de la arcilla (sobre todo micas blancas), fenómeno más acentuado cuando estos granos de feldespato coinciden con un borde de poro.

Con objeto de caracterizar la costra de oxalatos encontrada en la portada, se ha preparado una lámina delgado-pulida de una muestra que conservaba parte de la costra y parte de la piedra, para observar microscopicamente la estratigrafía, obtener información de la textura de la costra y de la interfase con la piedra.

Al microscopio se observa que hay una pelicula de espesor variable, de media aproximada de $0,5-1 \mathrm{~mm}$, de color rojizo-ocre con nicoles paralelos, que recubre todo el material pétreo, bastante compacta y de textura micrítica. La porosidad de ésta pelicula es mucho menor que la de la piedra, de modo que al salir hacia la superficie exterior la humedad con sales disueltas, se encuentra con esta barrera, provocando que dichas sales precipiten en la interfase piedra-costra. La acción mecánica de estas sales provoca que la costra se vaya desprendiendo, haciéndolo al principio sólo por las partes más débiles (figura 9), pero provocando al final el desprendimiento completo de la misma, que a la vez arrastra parte del material pétreo debilitado. Esto hace que la piedra quede desnuda, expuesta al exterior con una superficie irregular y descohesionada, acelerando su alteración y pérdida de material.

\subsection{Análisis Químico}

La preparación de las muestras se ha realizado mediante fusión alcalina y posterior extracción en medio ácido. Las determinaciones cuantitativas se han
The stone could be clasified as a micritic biocalcarenite with facies of bentonic and planctonic foraminifera, with great abundance of terrigenous; facies tipycal from medium Subbético and origin on bowl edge.

Porosity is very high, and possibly has been incremented with respect to the original on the quarry, by the disolution of Globotruncanes cameras. Pores are well conected, fact that facilitates circulation of fluids inside the stone.

It can be observed recrystallization of micritic calcium carbonate on the edges of pores, which indicates that, effectively, it has been taken place circulation of water that has dissolved the original carbonate that precipitates on pores after.

Feldspar terrigenous clastics appear quite alterated given phases more hydrated, as clay minerals (over all white micas), phenomenon more accentuated when feldspar grains coincide with pore edges.

To characterize oxalate crust find on the stone and detected by X Ray difraction, a thin section has been prepared of a sample with crust, to observe it microscopically.

It can be seen that there is a layer of variable thickness of 0,5-1 $\mathrm{mm}$ and reddish-ocre colour with plane polarized light, that covers great zones of the stone, quite compact and of micritic texture. Porosity of this layer is quite smaller than this of the stone, so, when water with disolved salts goes toward the surface, finds this barrier, causing that the salts precipitate on the interphase stone-crust. Mechanical action of these salts provoques crust separation and fall, first on weekest parts (see figure 9), and then the complete detachment of crust on greater zones, carrying away part of stone. In this way, stone becomes with a irregular surface and decohesionated, which accelerates its weathering and loss of material.

\subsection{Chemical Analysis}

Preparation of samples has been made by means of alcaline fussion and extraction on acid mean. Quantitative determination has been carried out by 


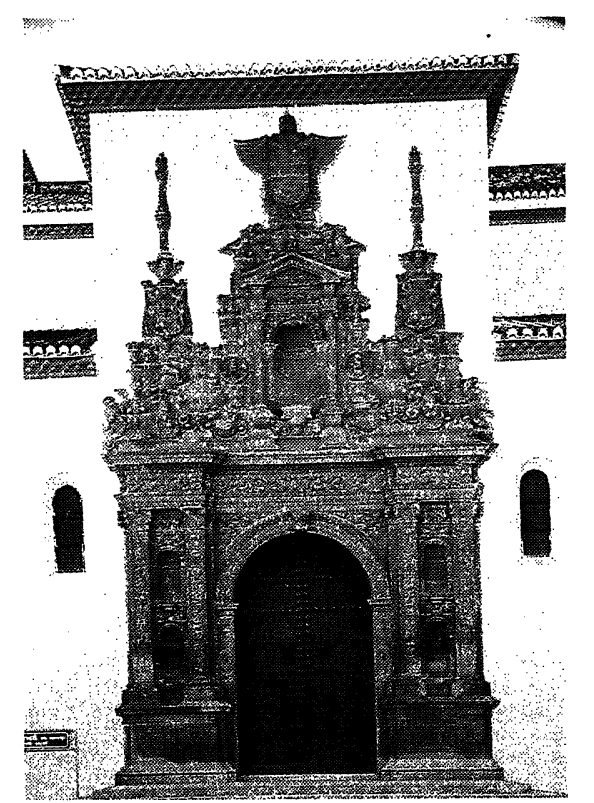

Fig. 1.- Portada de la iglesia de Santiago. lig. l. - Muin I )oor of Santiago church.

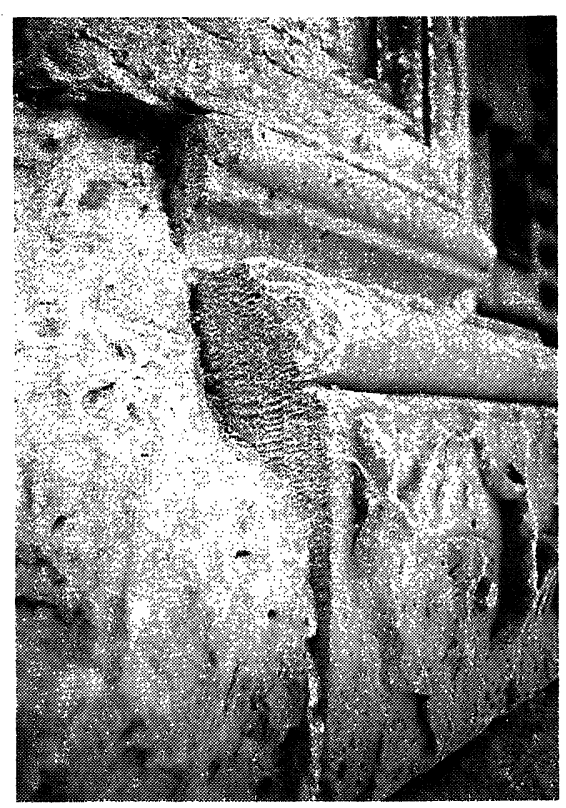

Fig.4.- Fenómenos de arcnización.

lig.t.- Arenization.

efectuado utilizando las técnicas de espectrofotometría de absorción UV-Vis, espectrofotometria de absorción atómica y valoración complexométrica. Los resultados obtenidos se recogen en las Tablas 2 y 3 .
I.T'- T'is espectrophotometry, atomic absorption espectrophotometry and complexometric titration. Results are given on Tables 2 and 3.
Fig. 2.- Nivel de humedad debido a la ascensión capilar. lig. 2.- Raising l amp Level.

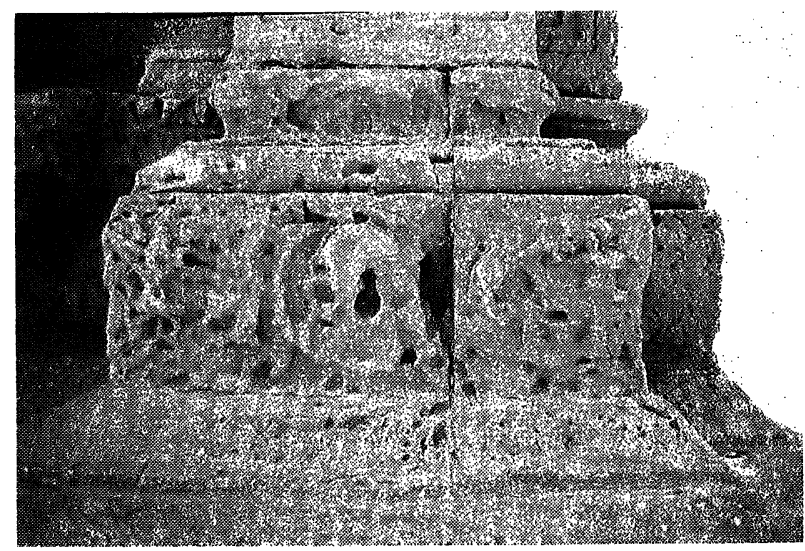

Fig.3.- Alveolización presente en la parte inferior izquierda de la portada.

Fig.3.- Alveolization on the main door left lower part.

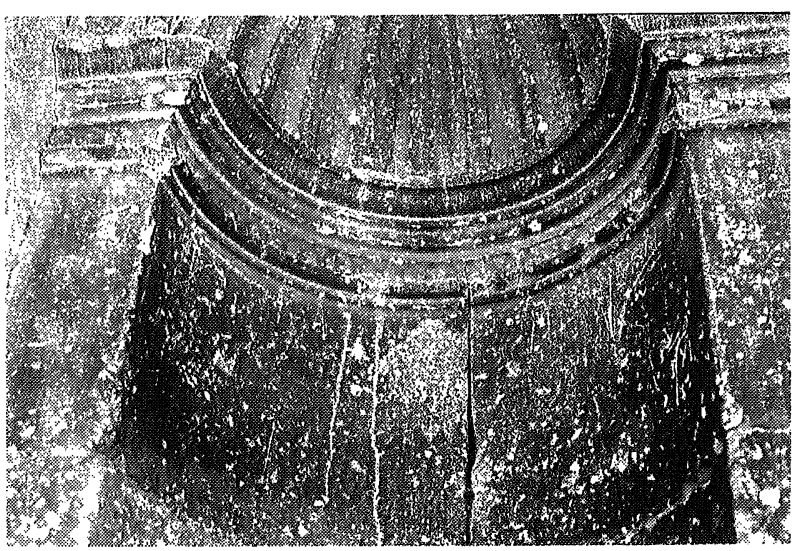

Fig.5.- Detalle de la costra oscura en la hornacina izquierda. lig. 5.-1)ark crust detail on the left niche. 


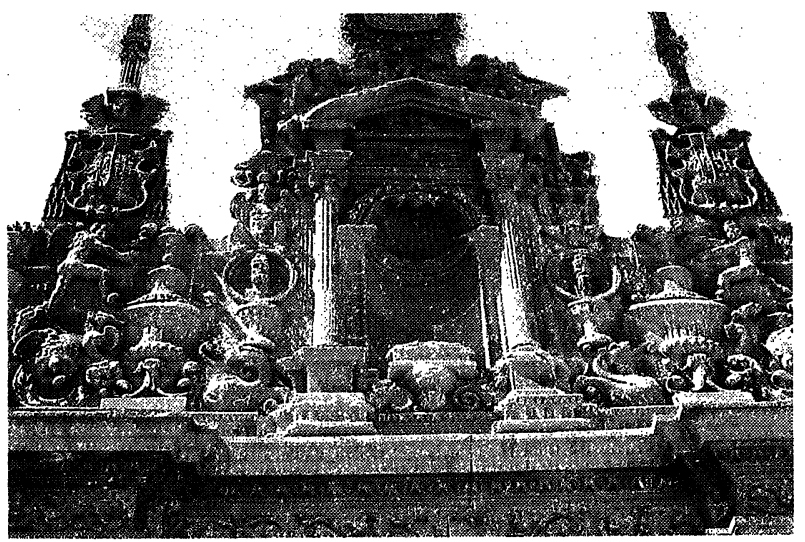

Fig.6.- Parte superior de la portada. lig. 6. - Main door upper part.

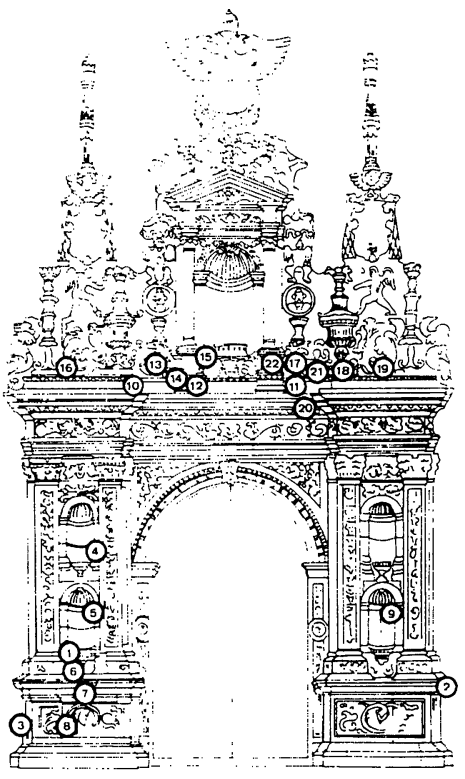

Fig. 7.- Localización de las muestras. lig. 7. - Samples location.

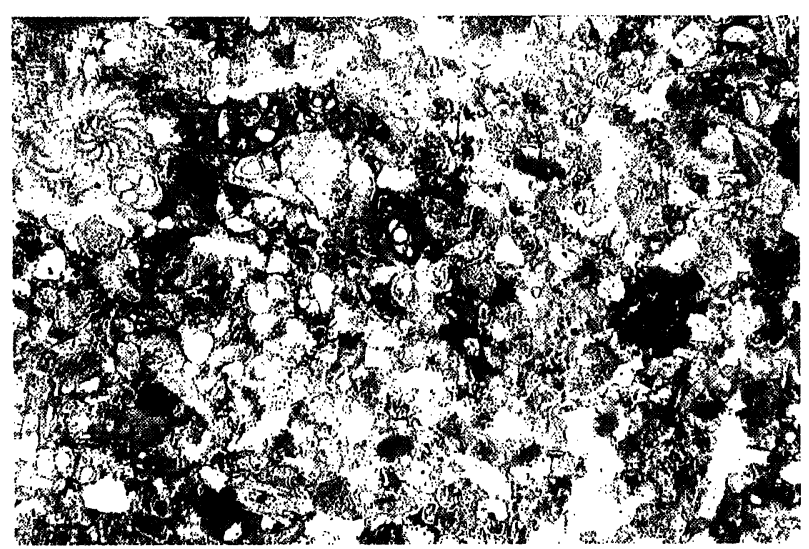

Fig.8.- Microfotografía de la piedra de la portada (x 40). lig.8.- Thin section microphotography of main door stone $(x+0)$.

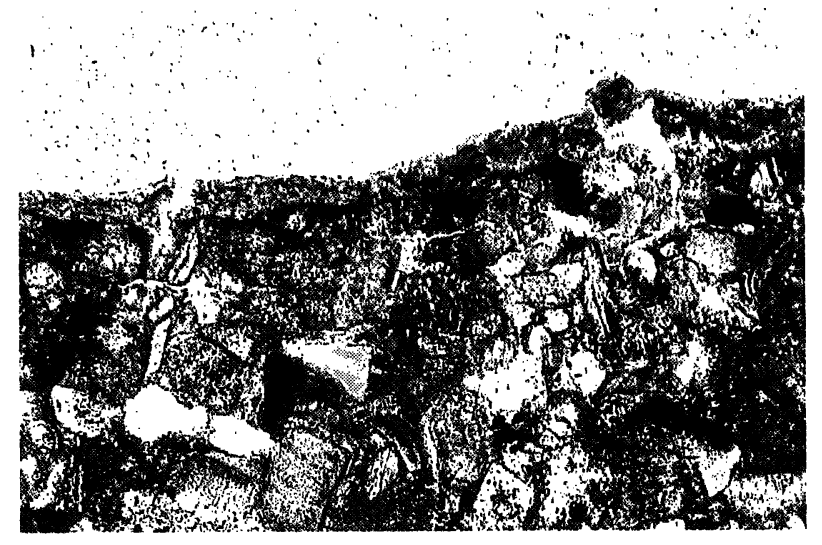

Fig.9.- Detalle al microscopio óptico de la costra oscura (x 100). Fig. 9. - Optical microscopy detail of the dark crust $(x 10)(0)$.

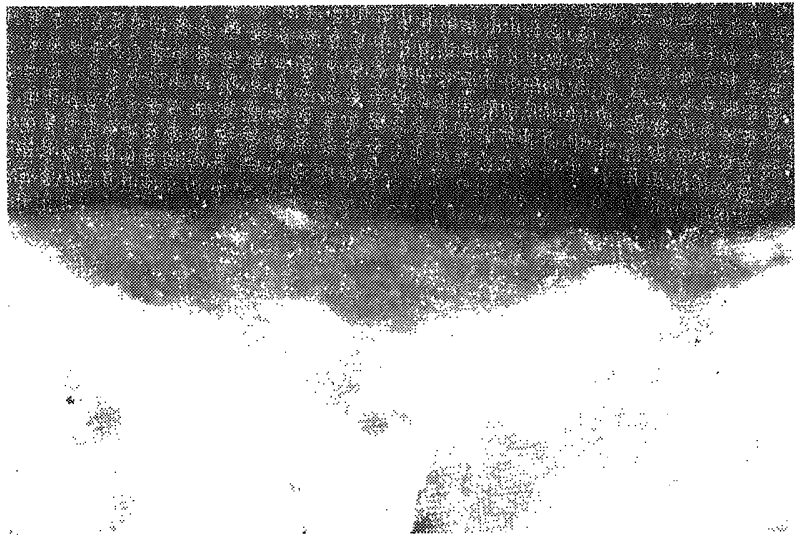

Fig. 10.- Estratigrafia de la capa pictórica. l'ig. 10. - Paint layer stratigraphy.

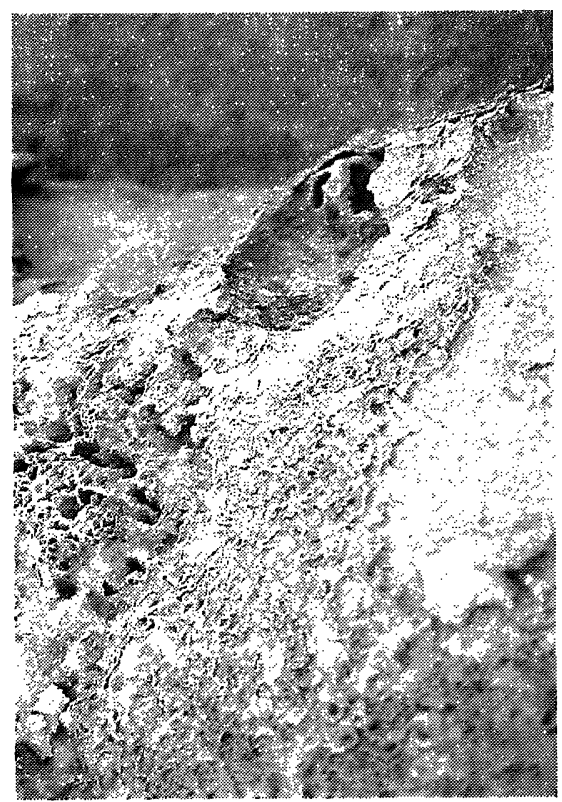

Fig. 11.- Ejemplo del grado de alteración encontrado en algunas zonas de la cantera.

Fig. 11.- Example of the alteration degree that appears on some zones of the quarry. 
TABLA 2 (TABLE 2)

Composición elemental cuantitativa (\%)

(Elemental quantitative composition (\%))

\begin{tabular}{||c|c|c|c|c|c|c|c|}
\hline $\begin{array}{c}\text { Muestra } \\
\text { (Samp/e) }\end{array}$ & $\begin{array}{c}\text { Pérdida } \\
\text { (Loss) }\end{array}$ & $\mathrm{SiO}_{2}$ & $\mathrm{CaO}$ & $\mathrm{MgO}$ & $\mathrm{Fe}_{2} \mathrm{O}_{3}$ & $\mathrm{Al}_{2} \mathrm{O}_{3}$ & $\mathrm{SO}_{3}$ \\
\hline \hline GPS1 & 37,78 & 14,53 & 38,11 & 1,95 & 0,92 & 1,08 & 0,68 \\
\hline GPS2 & & 12,40 & 39,60 & 2,22 & 0,79 & 1,01 & 1,77 \\
\hline GPS3 & 25,82 & 34,87 & 22,72 & 2,71 & 3,27 & 4,54 & 1,62 \\
\hline GPS4 & & 17,06 & 35,66 & 2,02 & 1,49 & 2,11 & 2,77 \\
\hline GPS5 & & 20,16 & 34,75 & 2,00 & 1,61 & 2,10 & 3,27 \\
\hline GPS6 & 31,02 & 30,94 & 28,65 & 2,60 & 2,40 & 2,70 & 1,37 \\
\hline GPS7 & & 4,78 & 37,32 & 0,58 & 0,28 & 0,28 & 31,75 \\
\hline
\end{tabular}

TABLA 3 (TABLE 3)

Composición química (\%)

(Chemical composition (\%))

\begin{tabular}{||c|c|c|c|c||}
\hline $\begin{array}{c}\text { Muestra } \\
\text { (Samp/e) }\end{array}$ & $\begin{array}{c}\text { Calcita } \\
\text { (Calcite) }\end{array}$ & $\begin{array}{c}\text { Cuarzo } \\
\text { (Quartz) }\end{array}$ & $\begin{array}{c}\text { Dolomita } \\
\text { (Dolomite) }\end{array}$ & $\begin{array}{c}\text { Yeso } \\
\text { (Gypsum) }\end{array}$ \\
\hline \hline GPS1 & 62,40 & 14,53 & 8,85 & 1,38 \\
\hline GPS2 & 63,00 & 12,40 & 10,14 & 3,78 \\
\hline GPS3 & 31,90 & 34,87 & 12,35 & 3,44 \\
\hline GPS4 & 55,20 & 17,06 & 9,24 & 6,02 \\
\hline GPS5 & 53,00 & 20,16 & 9,21 & 7,05 \\
\hline GPS6 & 43,00 & 30,94 & 11,98 & 2,92 \\
\hline GPS7 & 25,50 & 4,78 & 2,58 & 68,28 \\
\hline
\end{tabular}

\subsection{Análisis Minerálogico mediante Difracción de Rayos X}

Los resultados obtenidos por este método de análisis se recogen en la Tabla 4.

\subsection{Estudio de la Capa Pictórica}

Ante la existencia de costras que presentaban la apariencia de una posible capa pictórica, se han preparado varias muestras de costras de distintos colores con la técnica utilizada para el estudio de muestras de pintura. Posteriormente se han estudiado al microscopio

\subsection{Ray Difraction Mineralogical Analysis}

Results obtained with this tecnique are given on Table 4.

\subsection{Paint Layer Study}

Due to the presence of superficial layers with appareance of paint, samples of layers of several colours have been prepared on the same way that paint samples. Then, they have been observed with optical microscope and SEM, and only one type of layer has 
TABLA 4 (TABLE 4)

Composición mineralógica semicuantitativa (\%)

(Semiquantitative mineralogical composition (\%))

\begin{tabular}{|c|c|c|c|c|c|}
\hline $\begin{array}{l}\text { Muestra } \\
\text { (Sample) }\end{array}$ & $\begin{array}{c}\text { Calcita } \\
\text { (Calcite) }\end{array}$ & $\begin{array}{l}\text { Cuarzo } \\
\text { (Quartz) }\end{array}$ & $\begin{array}{l}\text { Dolomita } \\
\text { (Dolomite) }\end{array}$ & $\begin{array}{c}\text { Yeso } \\
\text { (Gypsum) }\end{array}$ & $\begin{array}{c}\text { Otros } \\
\text { (Others) }\end{array}$ \\
\hline GPS1 & 71 & 17 & 10 & & $\begin{array}{l}\text { Feldesp. } \\
\text { (Feldspar) }\end{array}$ \\
\hline GPS2 & 58 & 15 & 20 & $<5$ & $\begin{array}{l}\text { Feldesp. } \\
\text { (Feldspar) }\end{array}$ \\
\hline GPS3 & 38 & 20 & 5 & $\begin{array}{c}\text { trazas } \\
\text { (traces) }\end{array}$ & (1) \\
\hline GPS4 & * & * & $*$ & $\begin{array}{c}\text { trazas } \\
\text { (traces) }\end{array}$ & (2) \\
\hline GPS5 & $*$ & $*$ & $*$ & $\begin{array}{c}\text { trazas } \\
\text { (traces) }\end{array}$ & (2) \\
\hline GPS6 & 41 & 25 & $\begin{array}{l}\text { trazas } \\
\text { (traces) } \\
\end{array}$ & & $\begin{array}{l}\text { Arcilla } \\
\text { (Clay) }\end{array}$ \\
\hline GPS7 & 52 & $<5$ & & 40 & Anhidrita. \\
\hline GPS8 & 66 & 14 & 8 & & $\begin{array}{l}\text { Feldesp. } \\
\text { (Feldspar) }\end{array}$ \\
\hline GPS11 & 69 & 20 & 11 & & $\begin{array}{c}\text { Oxalato } \\
\text { (Oxalate) }\end{array}$ \\
\hline GPS12 & * & * & & & $\begin{array}{c}\text { Oxalato } \\
\text { (Oxalate) }\end{array}$ \\
\hline GPS13 & 64 & 13 & 15 & & $\begin{array}{l}\text { Arcilla } \\
\text { (Clay) }\end{array}$ \\
\hline GPS15 & 27 & 17 & $<5$ & & $\begin{array}{l}\text { Arcilla } \\
\text { (Clay) }\end{array}$ \\
\hline GPS18 & 30 & 24 & $<5$ & & $\begin{array}{l}\text { Arcilla } \\
\text { (Clay) }\end{array}$ \\
\hline GPS20 & 43 & 15 & 17 & & $\begin{array}{l}\text { Arcilla } \\
\text { (Clay) }\end{array}$ \\
\hline
\end{tabular}

(1) Feldespatos, arcilla y oxalatos / Feldspars, clay and oxalates

(2) Arcillas y oxalatos / Clays and oxalates

* No es posible calcular proporción por la presencia de minerales de los que no se tienen datos suficientes / The proportion cannot be calculated owing to the lack of data on certain minerals present.

óptico y electrónico, y tan sólo una de ellas se ha identificado como capa aplicada artificial e intencionadamente. La estratigrafia de dicha muestra al microscopio aparece en la figura 10 , donde se aprecia una capa de color marrón, aplicada directamente sobre la piedra y formada por pigmentos a base de compuestos de hierro, como se detecta en el microanálisis efectuado con la microsonda de Rayos X. been identified as artificial layer. The stratygraphy of this sample appears on figure 10, where it could be seen a brown-reddish layer, applied directly over the stone and composed by pigments based on iron compounds, as it is detected with SEM-EDT. 


\section{CONSIDERACIÓN DE RESULTADOS}

En las Tablas 5 a 7 se recoge la composición de las muestras agrupadas por tipo de material.

En todas las muestras analizadas aparecen como componentes mayoritarios la calcita, la dolomita y la sílice, con pequeños porcentajes de yeso, por lo que puede confirmarse la existencia de un único tipo de piedra.

\section{EVALUATION OF RESULTS}

On Tables 5 to 7 is shown the composition of samples of each type of material.

All the stone samples analyzed have as main components calcite, dolomite and quartz, with little quantities of gypsum; it can be confirmed that only one type of stone has been used on the door front.

TABLA 5 (TABLE 5)

Composición de muestras de piedra (Composition of stone samples)

\begin{tabular}{|c|c|c|c|c|}
\hline $\begin{array}{c}\text { Muestra } \\
\text { (Samp/e) }\end{array}$ & $\begin{array}{c}\text { Calcita } \\
\text { (Calcite) }\end{array}$ & $\begin{array}{c}\text { Cuarzo } \\
\text { (Quartz) }\end{array}$ & $\begin{array}{c}\text { Dolomita } \\
\text { (Dolomite) }\end{array}$ & $\begin{array}{c}\text { Yeso } \\
\text { (Gypsum) }\end{array}$ \\
\hline \hline GPS1(X) & 71 & 17 & 10 & 1,38 \\
\hline GPS1(q) & 62,40 & 14,53 & 8,85 & $<5$ \\
\hline GPS2(X) & 58 & 15 & 20 & 3,78 \\
\hline GPS2(q) & 63,00 & 12,40 & 10,14 & 8 \\
\hline GPS8(X) & 66 & 14 & 11 & 15 \\
\hline GPS11(X) & 69 & 20 & 13 & \\
\hline GPS13(X) & 64 & 15 & & \\
\hline GPS20(X) & 43 & & & \\
\hline
\end{tabular}

(X) Resultados Difracción de Rayos X / X Ray Difraction results

(q) Resultados análisis químico / Chemical analysis results

TABLA 6 (TABLE 6)

Composición de muestras de morteros (Composition of mortar samples)

\begin{tabular}{||c|c|c|c|c|}
\hline $\begin{array}{c}\text { Muestra } \\
\text { (Samp/e) }\end{array}$ & $\begin{array}{c}\text { Calcita } \\
\text { (Calcite) }\end{array}$ & $\begin{array}{c}\text { Cuarzo } \\
\text { (Quartz) }\end{array}$ & $\begin{array}{c}\text { Dolomita } \\
\text { (Dolomite) }\end{array}$ & $\begin{array}{c}\text { Yeso } \\
\text { (Gypsum) }\end{array}$ \\
\hline GPS6(X) & 41 & 25 & $<5$ & 2,92 \\
\hline GPS6(q) & 43,00 & 31,02 & 11,98 & $<5$ \\
\hline GPS15(X) & 27 & 17 & $<5$ & \\
\hline GPS18(X) & 30 & 24 & & $<$ \\
\hline
\end{tabular}

(X) Resultados Difracción de Rayos X / $X$ Ray Difraction results

(q) Resultados análisis químico / Chemical analysis results 
TABLA 7 (TABLE 7)

Composición de muestras de costra y eflorescencias

(Composition of crust and eflorescences samples)

\begin{tabular}{||c|c|c|c|c||}
\hline $\begin{array}{c}\text { Muestra } \\
\text { (Samp/e) }\end{array}$ & $\begin{array}{c}\text { Calcita } \\
\text { (Calcite) }\end{array}$ & $\begin{array}{c}\text { Cuarzo } \\
\text { (Quartz) }\end{array}$ & $\begin{array}{c}\text { Dolomita } \\
\text { (Dolomite) }\end{array}$ & $\begin{array}{c}\text { Yeso } \\
\text { (Gypsum) }\end{array}$ \\
\hline GPS3(X) & 38 & 20 & 5 & $\begin{array}{c}\text { trazas } \\
\text { (traces) }\end{array}$ \\
\hline GPS3(q) & 31,90 & 34,87 & 12,35 & 3,44 \\
\hline GPS4(X) & $*$ & $*$ & $*$ & $\begin{array}{c}\text { trazas } \\
\text { (traces) }\end{array}$ \\
\hline GPS4(q) & 55,20 & 17,06 & 9,24 & 6,02 \\
\hline GPS5(X) & $*$ & $*$ & $*$ & trazas \\
(traces)
\end{tabular}

(X) Resultados Difracción de Rayos X / X Ray Difraction results

(q) Resultados análisis químico / Chemical analysis results

GPS7 Eflorescencias / Eflorescences

Los morteros utilizados son todos de cal y arena, con pequeñas cantidades de dolomita y yeso, que podrían proceder de la piedra por migración al ser compuestos más solubles.

Las costras básicamente tienen la misma composición que la piedra, observándose un enriquecimiento en yeso y la presencia de oxalato cálcico (determinado por la Difracción de rayos $\mathrm{X}$ ). La muestra de eflorescencias analizada tiene un contenido mucho más alto en yeso.

\section{LOCALIZACIÓN DE LA CANTERA}

Con objeto de caracterizar el material pétreo inalterado y obtener suficiente cantidad de muestras (probetas cúbicas de $5 \mathrm{~cm}$ de arista) para la realización de los ensayos necesarios, con el fin de evaluar los posibles tratamientos de restauración, se ha procedido a localizar la cantera de donde se extrajo la piedra. Esta procedencia está suficientemente documentada, ya que es una de las canteras más importantes de la Catedral de Guadix.

Tras realizar unos análisis de comprobación a partir de muestras proporcionadas por Alcalde (2), se efectuó la
The mortars used are all formed by lime and sand (calcite and quartz) with little quantities of dolomite and gypsum, that could procede by migration from the stone due to their more elevated solubility.

Crusts basically have the same composition than the stone, with and enrichment on gypsum and the presence of calcium oxalate (detected by X Ray Difraction). The efflorescence sample analyzed has a very high content of gypsum.

\section{QUARRY LOCALIZATION}

With the aim of characterize unweathered stone and to get enough quantity of samples (cubes of $5 \mathrm{~cm}$ ) to carry out all the tests needed to evaluate possible treatment products, we have proceded to find the quarry from where stone was extracted. There is enough documentation about it, as this is one of the more important quarries used on the Cathedral of Guadix.

After analyzing a sample of the quarry given by $M$. Alcalde (2) to verify its coincidence, the extraction of 
toma de muestras y extracción de bloques de la cantera para proceder a la preparación de las probetas. Las muestras analizadas se recogen en la Tabla 8. blocks from the quarry was effected to prepare cubic samples. The analysis of a sample is given on Table 8.

TABLA 8 (TABLE 8)

Composición de piedra de cantera

(Composition of quarry stone)

\begin{tabular}{|c|c|c|c|c|c||}
\hline $\begin{array}{c}\text { Muestra } \\
\text { (Samp/e) }\end{array}$ & $\begin{array}{c}\text { Calcita } \\
\text { (Calcite) }\end{array}$ & $\begin{array}{c}\text { Cuarzo } \\
\text { (Quartz) }\end{array}$ & $\begin{array}{c}\text { Dolomita } \\
\text { (Dolomite) }\end{array}$ & $\begin{array}{c}\text { Yeso } \\
\text { (Gypsum) }\end{array}$ & $\begin{array}{c}\text { Otros } \\
\text { (Others) }\end{array}$ \\
\hline \hline CB1(X) & 70 & 12 & 12 & & $\begin{array}{c}\text { Feldesp. } \\
\text { (Feldspar) }\end{array}$ \\
\hline CB1(q) & 67,20 & 14,28 & 8,64 & 1,21 & \\
\hline
\end{tabular}

(X) Resultados Difracción de Rayos $X / X$ Ray Difraction results

(q) Resultados análisis químico / Chemical analysis results

Como puede observarse comparando los resultados de las muestra de piedra de la portada con los de la cantera, los valores de los componente mayoritarios son muy semejantes; además, se comprueba que el pequeño contenido en yeso de varias muestras de la portada coincide con el detectado en la piedra de cantera. De igual modo la comparación petrográfica también nos indica que se trata del mismo material.

La cantera se encuentra en Bácor, pequeña localidad situada a unos $40 \mathrm{~km}$ al Noreste de Guadix, concretamente a 1 ó $2 \mathrm{~km}$ al Norte de dicha población, siguiendo el curso ascendente de la rambla del Baul. La vía de comunicación entre Guadix y dicha población es bastante buena, aunque el acceso concreto a la cantera es más difícil por encontrarse en lugar escarpado y abandonado.

Geológicamente se encuentra dentro de la denominada Depresión Guadix-Baza, cuya edad abarca desde el Oligoceno Superior hasta el Cuaternario, perteneciente al Subbético Medio. Concretamente está inmersa dentro de un paquete datado como Mioceno Inferior-Medio, compuesto de calcarenitas, margas y calizas.

Geomorfológicamente se encuentra en la ladera izquierda (en sentido ascendente) de un pequeño valle socavado por el río que transcurre por el mismo, que ha generado paredes muy escarpadas y donde los fenómenos de "karstificación" han sido bastante pronunciados. Esto ha propiciado que los afloramientos de este material calcarenítico en este lugar sean muy abundantes y de fácil extracción en grandes cantidades.
Comparing the results of stone samples from the door front with that from the quarry, it can be seen that the values of main components are very similar; also the mileralogical characteristics of both stones are the same. In adition, it is confirmed that the little content of gypsum that appears on several samples from the door front coincides with that detected on stone from the quarry.

The quarry is located near Bácor, little village situated $40 \mathrm{Km} N E$ from Guadix, more exactly 1 or $2 \mathrm{Km}$ to the North of that town following the upward course of the watercourse Baul. The road between Guadix and Bácor is quite good, although the acces to the quarry is more difficult as it is located on a steep and deserted place.

Geologically it is situated on the so called GuadixBaza depression, which age comes from Superior Oligocene to Cuaternary, belonging to Middle Subbético. Exactly it is inmersed on a zone dated as Inferior-Middle Miocene, composed by calcarenite, marls and limestones.

Geomorphologically, the quarry is on the left slope (on ascendent sense) of a little valley hollowed by the river that passed by it, that has formed very steep walls and where karstification phenomena have been very important. These facts have propitiated that the outcrops of this calcarenite are very numerous and easily extractable on great quantities. 
Los fenómenos de alteración encontrados en algunos lugares del afloramiento llegan a ser incluso más graves que en la propia portada. Como formas de alteración se han encontrado alveolización y arenización muy pronunciadas, así como desplacaciones y escamaciones de material de suma importancia (figura 11). También se han encontrado grandes costras de recristalización de carbonatos y zonas donde las eflorescencias recubren todo el material. En definitiva, fenómenos íntimamente relacionados con la disolución y recristalización, dando formas de alteración ligadas con la karstificación vigente y que es aún más propiciada por el enclave y morfología del afloramiento.

\section{CONCLUSIONES}

Con los resultados analíticos obtenidos se puede afirmar que la piedra de la portada de la Iglesia de Santiago procede de la cantera de Bácor que, asimismo, fue empleada en la Catedral de Guadix. Ya en cantera la piedra presenta un pequeño contenido en sulfato, que forma parte de sales solubles que contribuyen a la alteración del material, tanto en cantera como en el edificio.

La costra oscura que cubre zonas muy amplias de la portada está formada básicamente por oxalato cálcico, compuesto que, según diversos autores, puede deberse a la reacción de un antiguo tratamiento a base de productos orgánicos con el carbonato cálcico de la piedra, con o sin la concurrencia de determinadas bacterias. Este tratamiento cubriría originalmente toda la portada, por lo que la costra, en su inicio, tendría esa misma extensión, desprendiéndose debido a los factores de alteración que inciden sobre la portada.

Adicionalmente se ha determinado la presencia de una película pictórica en algunas áreas muy concretas de la portada, sólo se ha podido identificar un único color, rojizo, a base de compuestos de hierro. Existen referencias (3) de que la portada estuvo policromada, aunque no se especifica si de uno o varios colores.

Del estudio de los indicadores visuales de alteración, las características de las costras, y las condiciones ambientales de la portada, se pueden determinar las causas del deterioro del material pétreo. El alto nivel freático del subsuelo favorece la subida de agua por capilaridad, la cual lleva disueltas diversas sales y disuelve algunos componentes de la piedra (especialmente los sulfatos). Con los cambios de temperatura y HR externas se produce el movimiento de la solución por el interior de la piedra, donde pueden producirse diversas reacciones entre ellos y con los componentes de la piedra (básicamente con la calcita y la dolomita), y puede tener lugar la cristalización de
The alteration indicators that appear on some zones of the outcrop are even more important that those found on the door front. There are alveolization and arenization very pronounced, and also very important desplacaciones and scaling out of material (figure 11). There are also crusts of carbonates recrystallized and zones where eflorescences cover completely the stone. All these phenomena are related to the disollution and recrystallization, that give alteration indicators related to karstification.

\section{CONCLUSIONS}

With the analytical results obtained it is possible to affirm that the stone from the door front of Santiago Church comes from Bácor quarry, that was used also on the Cathedral of Guadix. Even in the quarry the stone presents a little content of sulfate, which forms part of soluble salts which contribute to the alteration of the material, both on the quarry and on the building.

Dark crust that covers wide areas of the door front is formed mainly by calcium oxalate, that could be originated by the reaction of an ancient treatment based on organic substances with calcite of the stone, with or without the concurrence of some bacteria. This treatment originally would cover all the door front, for what the crust initially would have the same extension and has going falling due to the weathering factors that affect the door front.

Besides, the presence of a paint layer on some areas has been determined, with only one colour identified, reddish, based on iron compounds. There are references (3) about the existence of polycromy on the door front, although it is not specified whether one or more colours.

From the study of visual weathering indicators, crusts characteristics and environmental conditions, it is possible to determine the causes of stone alteration. The high ground water level favours rasing damp, water which carries in solution several salts and disolves some components of stone (specially sulfates). With the changes in external temperature and $R H$, this sollution moves by the inner part of stone, where the soluble salts can react between them and with other components of the stone (calcite and dolomite mainly), and the crystallization of some compounds can take place. Oxalate crust, that originally would cover the major part of the door front, is much more compact 
diversos compuestos. La costra de oxalatos, que originalmente cubriría gran parte de la portada, es mucho más compacta que la piedra subyacente, de forma que dificulta el paso del agua con sales disueltas que circulan por el interior de la piedra, produciéndose su acumulación detrás de ella. Al evaporarse el agua en la superficie, se produce la cristalización de los compuestos que lleve en solución detrás de la costra de oxalato; esto provoca la disgregación de la piedra debajo de dicha costra y termina provocando el desprendimiento de la misma. Cuando la piedra disgregada queda expuesta al exterior, la alteración progresa con rapidez, apareciendo los indicadores de alteración que ya se han mencionado.

Efectos parecidos son los causados por la formación de hielo en el interior de la piedra cuando las temperaturas descienden por debajo de $0^{\circ} \mathrm{C}$. Las diferentes porosidades de la costra y de la piedra hacen que los cambios de volumen debido al hielo causen la disgregación de la piedra y el desprendimiento de la costra.

El yeso detectado en la zona más externa de la piedra, costras y eflorescencias (3-7\%), con algunos valores puntuales muy elevados, puede tener su origen en parte en mecanismos de alteración relacionados con la contaminación atmosférica, y en parte en el yeso existente en la propia piedra. Al ser un compuesto más soluble que los carbonatos, se produce su migración hacia el exterior y el enriquecimiento del mismo en la superficie de la piedra. that subjacent stone, so it difficults the pass of water with dissolved salts that circulate through the stone, and causes the accumulation of this solution behind it When water evaporates on the surface, salts crystallize behind the crust, causing stone disagregation and, finally, the detachment of the crust. When the stone disagregated becomes exposed to the environment, weathering progresses very quickly appearing alteration indicators already mentioned.

Similar effects are caused by frost formation in the interior of stone when temperatures drops below $0^{\circ} \mathrm{C}$. Diferent porosities of crust and stone make that volumes changes due to frost causes disagregation and detachment of crust.

Gypsum found on the surface of stone, crusts and efflorescences (3-7\%) with some values very high, could have its origin, by one side, on weathering mechanisms related with atmospheric pollution and, by other side, on gypsum pre-existing on the stone itself. Being a compound more soluble that carbonates it takes place its migration towards the exterior and its enrichment on stone surface.

\section{BIBLIOGRAFÍA}

(1) ASENJO SEDANO, C.: Guadix: Guía Histórica y Artística. Diputación Provincial de Granada. 1989.

(2) ALCALDE MORENO, M.; MARTIN, A.: Morfología Macroscópica de Alteración de la Piedra de la Catedral de Guadix/ España. Materiales de Construcción. Vol.41, N²24, 1991, pgs 21-41.

(3) GOMEZ-MORENO CALERA, J.M.: Un nuevo proyecto de Siloé: la iglesia de Santiago de Guadix. Cuadernos de Arte de Universidad de Granada. N XXIV, 1993, 21-39. 